\title{
Audio-Magnetotelluric Study of a Structure with a Reverse Fault
}

\author{
P. -A. SChNeGG*, B. V. Le QuANG*, G. FischeR*, \\ and J. T. WEAVER** \\ *Observatoire Cantonal, Neuchâtel, Switzerland \\ **Department of Physics, \\ University of Victoria, Victoria, B.C., Canada
}

(Received August 15, 1983)

\begin{abstract}
A two-dimensional (2-D) structural transition from a flat valley floor to a mountain slope, with a presumed reverse fault at its base, was investigated by the audio-magnetotelluric sounding method. By calling on previously available geological information, it was possible to reformulate the problem so that only two basic questions remained to be answered: (1) does the structure effectively involve a fault, and (2) if so what is the dip angle of this fault? Since the soundings away from the mountain furnished the 1-D valley structure, it was possible to construct a 2-D model in which the only specified parameter was the unknown dip angle. Even the absence of a fault could be simulated in the model by giving this parameter a definite value. The model responses were computed numerically by the technique of finite differences and compared with the measured response curves, yielding quite clear answers in favor of a reverse fault dipping at an angle of less than $30^{\circ}$ under the mountain. It was also possible to secure some information concerning details of the valley structure.
\end{abstract}

\section{Introduction}

The techniques of magnetotelluric (MT) and audio-magnetotelluric (AMT) sounding have often been employed in the study of horizontally layered structures, so-called one-dimensional (1-D) structures. But these methods have also proven their usefulness in the investigation of 2-D structures. The 2-D studies are generally conducted in terms of a series of soundings along a profile. In most instances each sounding site is then interpreted as a 1-D site, and a 2-D profile is obtained by joining related formations (see as an example STANLEY et al., 1977). In some recent investigations the profile derived in this fashion is considered as an initial model only, and is subsequently modified according to a 2-D modelling routine which attempts to fit the field data to a numerically derived model response. An example of such a procedure is given by HUTTON et al. (1980), the modelling routine being provided by BREWITT-TAYLOR and JOHNS (1980). Another 2-D inversion method for MT data has been proposed by JUPP and Vozoff (1977). But the task of inverting or modelling 2-D data remains 
rather formidable. The two schemes quoted, even if they may provide unequivocal inversion or modelling recipes, have not been tested from the point of view of the uniqueness of the solution they propose, or whether the fit they achieve is the best possible. In these respects the 2-D problem cannot be compared with the much simpler 1-D inversion (cf. e.g., FISCHER and LE QUANG, 1981, 1982).

Instead of directing our attention to the general question of interpreting 2-D data along a profile, we look at a very simple structure, whose overall geological features are well known already. An example will be given where AMT was capable of confirming and quantifying a structural feature for which there was, so far, only very indirect geological evidence, mainly because this is a buried feature in an area where no drilling has yet been undertaken.

\section{The Geological Setting}

In the course of building an AMT system (c.f., e.g., SCHNEGG and FISCHER, 1980, or FISCHER, 1982) many trial soundings were performed near our home base of Neuchâtel. In the vicinity of Neuchâtel two different types of formations are common. The first one consists of compact limestones in the Folded Jura, with high resistivities of $10^{3}-10^{4} \Omega \mathrm{m}$. The second kind are low resistivity sites, like those over the Swiss Molasse Basin with typical resistivities of $30 \Omega \mathrm{m}$, or sites in the Jura mountains where tertiary and quaternary sediments overlay the compact mesozoic limestones and yield apparent resistivities as low as 5-10 $\Omega \mathrm{m}$. We found it very difficult, if not totally impossible, to get good data in the high resistivity areas. This is not too surprising considering the great population density and the high level of industrialization of the country, for high resistivities imply large electric fields and great penetration depths and mean, therefore, that perturbations are carried far afield. An example of such a sounding is given in Fig. 7, where the wide data scatter is quite apparent. In the low resistivity areas it was far easier to obtain good data, even sometimes within close range of a village. Data from low resistivity sounding sites are shown in Figs. 4-6 and in Fig. 9.

In view of the possibility of obtaining what appeared to be rather good AMT data in the Val de Ruz, the obvious question was to ask whether these data were in accord with the geological information available about this area, shown in the map of Fig. 1. This map gives the location of a geological profile through the study area. This profile is reproduced in Fig. 2 and the information it contains is quite pertinent to the AMT profile, even though this second profile lies about one $\mathrm{km}$ further to the NE. It is worth noticing that on the geological profile the slope of the mountain is only about $17^{\circ}$ to $20^{\circ}$, whereas it is about $30^{\circ}$ to $35^{\circ}$ along the AMT profile. The profile of Fig. 2 is taken from the SwISS GEOLOGICAL ATLAS (1:25000 scale), which published the geological map and profile of the Val de Ruz in 1969. While the geology of the area is securely established in its broad outline, the detailed features of Fig. 2 are only conjectural. in fact there is indirect evidence that the transition between the valley floor (Val 


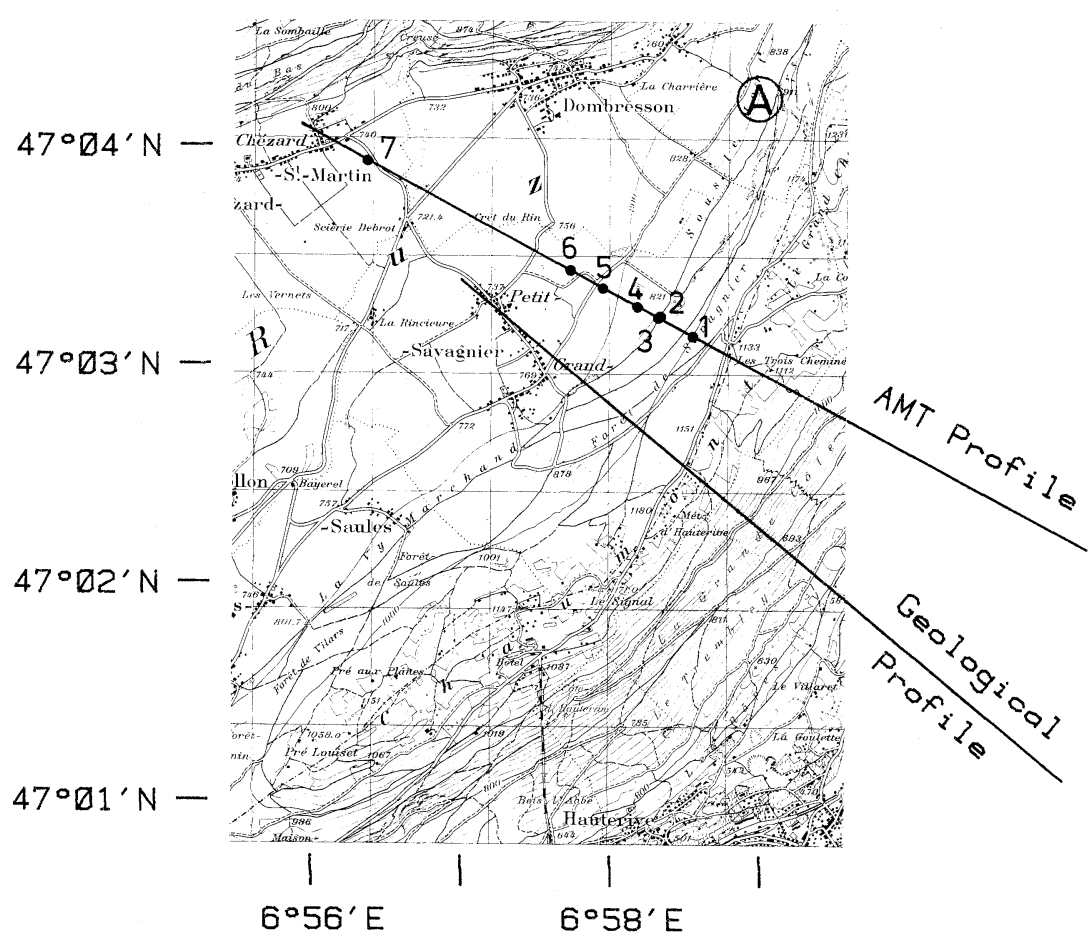

Fig. 1. Geographical map of the study area. The line through the centre of the village of PetitSavagnier corresponds to the geological profile of Fig. 2. The AMT profile gives the sounding sites 1 to 7 . The letter $A$ at top right is on a profile where a fault has been observed. Reproduced with permission from the Swiss Federal Office of Topography.

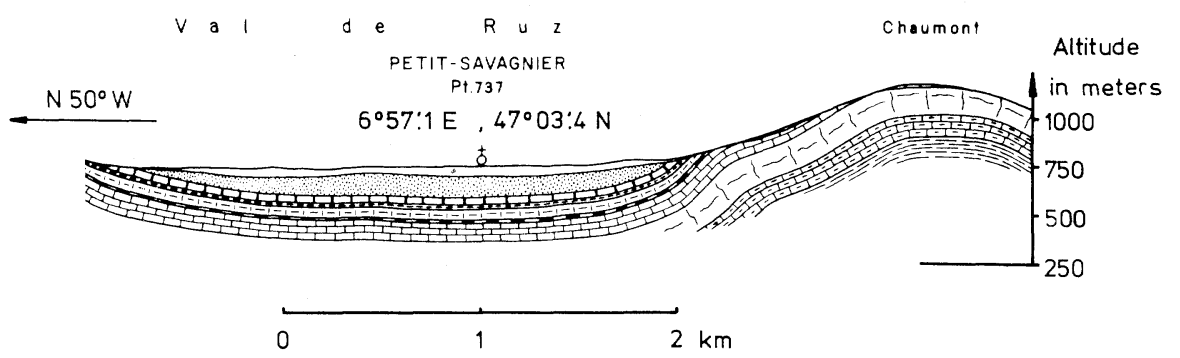

Fig. 2. Geological profile through Petit-Savagnier. The valley formations are, respectively and with increasing depth; Quaternary, Tertiary (sandstones), several Secondary formations (various mesozoic or cretaceous limestones). 


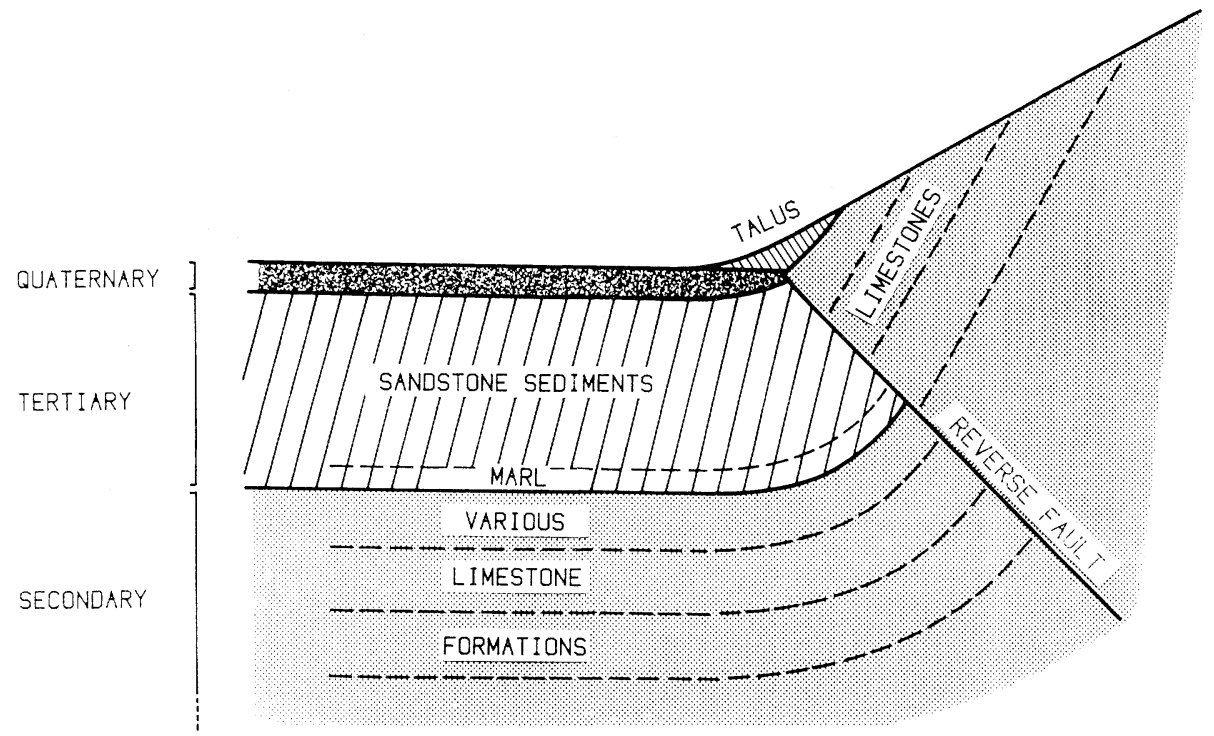

Fig. 3. Assumed geology along the AMT profile. The talus or scree hides the possible outcrop of the presumed fault, which may be a consequence of the lateral pressures responsible for the Jura folds.

de Ruz) to the slope of Chaumont involves a reverse fault as sketched in Fig. 3. Evidence in favor of the fault is obtained from geological features $3 \mathrm{~km}$ to the NE, where the absence of a screening talus reveals a slight fracture dipping with an angle of about $45^{\circ}$ at location A of the Fig. 1 map, but other geological differences prevent its simple continuation toward the profiles of Figs. 2 and 3. Not only is the presence or absence of a fault in the profiles of Figs. 2 and 3 not yet ascertained, but also unkown is the angle of $\operatorname{dip} \alpha$ of this fault, as well as the depth of the various formations to the NW of the mountain base. It is quite likely that the various secondary or mesozoic layers forming the valley floor are in fact dipping slightly as they approach the mountain, as suggested by the data from Fig. 10.

While the first soundings in the Val de Ruz were performed with the view to testing AMT equipment, it became gradually apparent that these soundings might prove capable of answering the questions formulated in the preceeding paragraph, that is (1) presence or absence of a fault, (2) angle of dip of this fault, (3) quantitative information regarding the valley formations in front of the fault, as well as their possible dip.

\section{AMT Data Acquisition and Processing}

The AMT equipment used has been described orally on several occasions, 

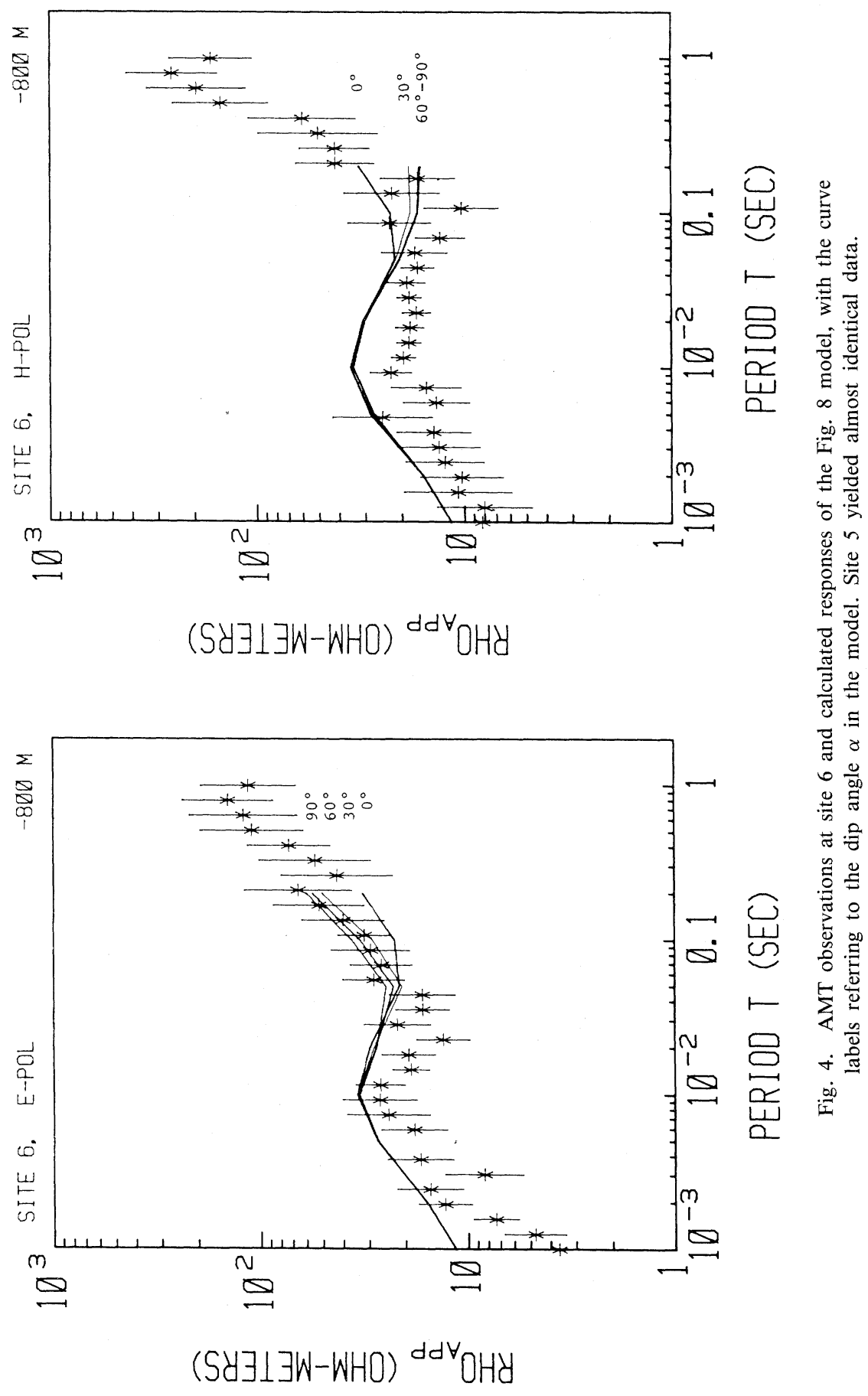

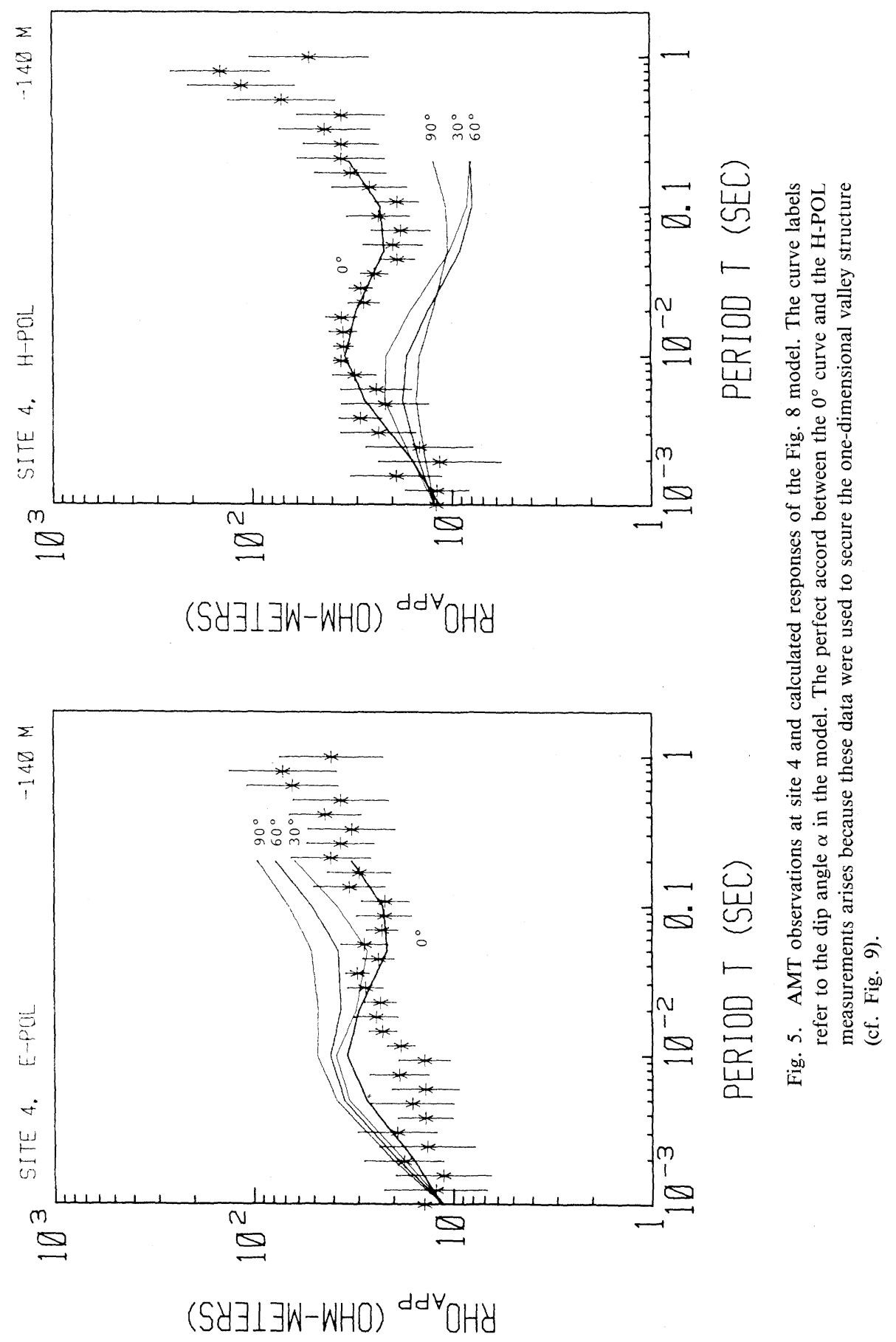


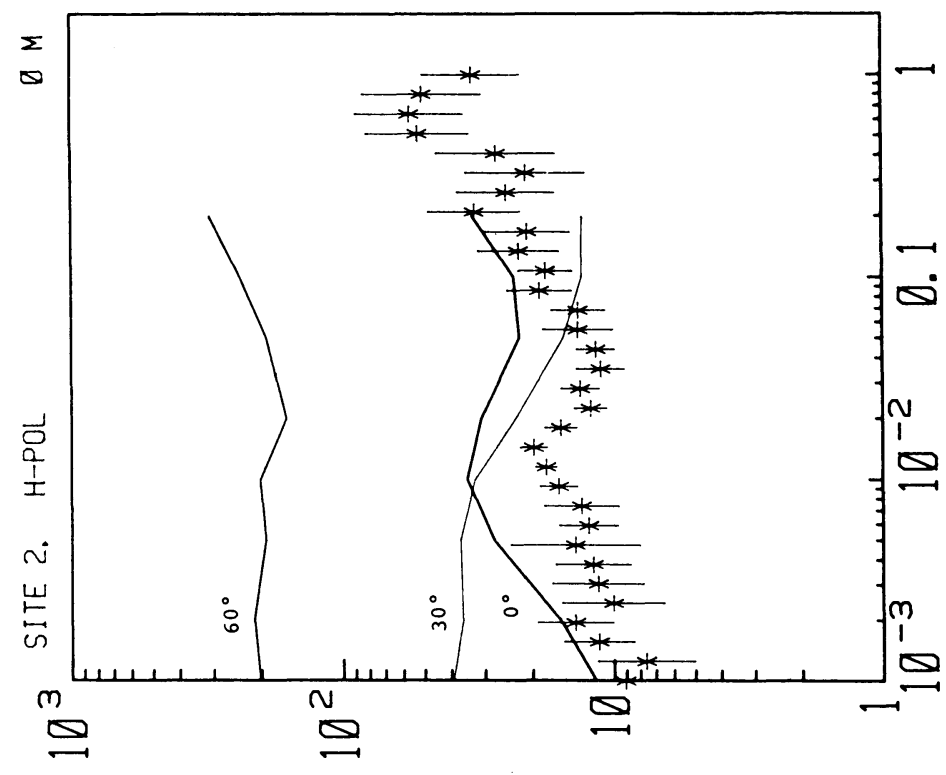

$(S Y \exists 1 \exists W-W H O){ }^{d d V} O H Y$

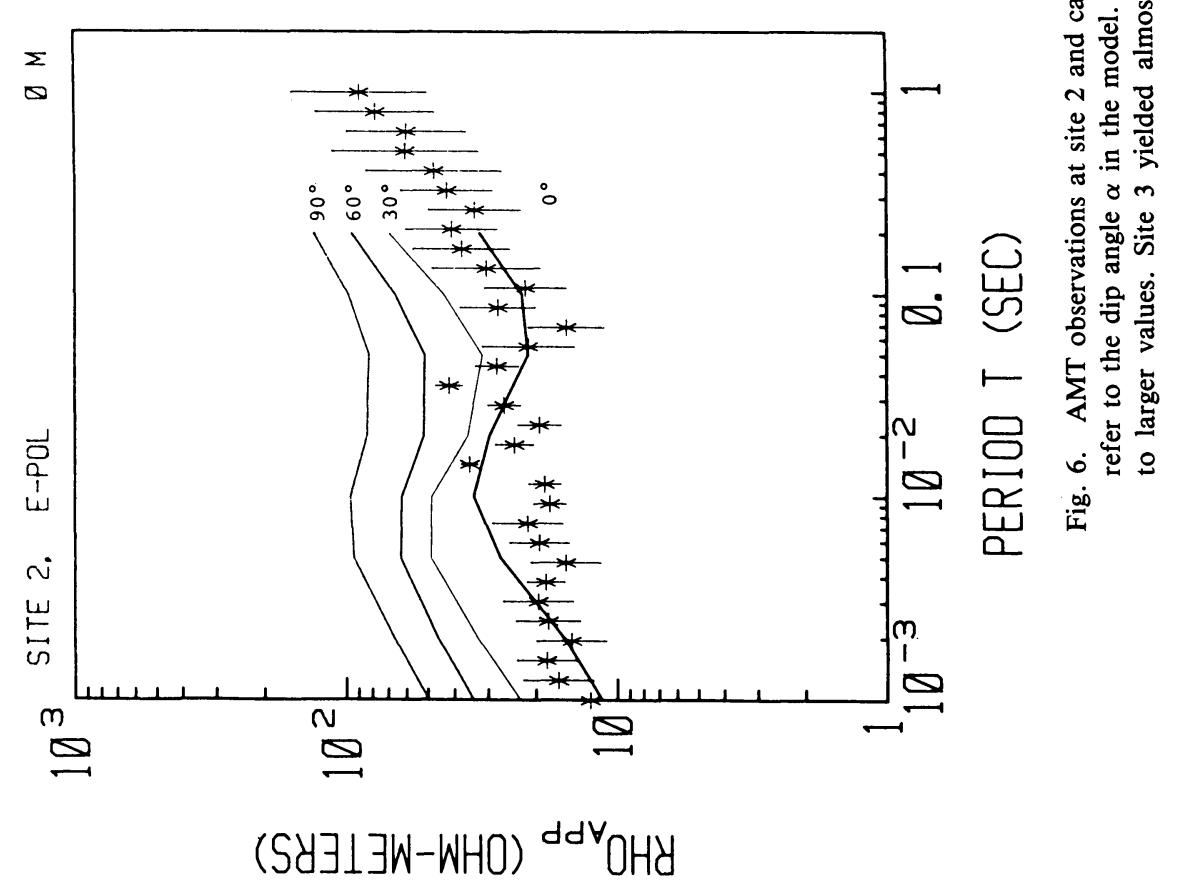




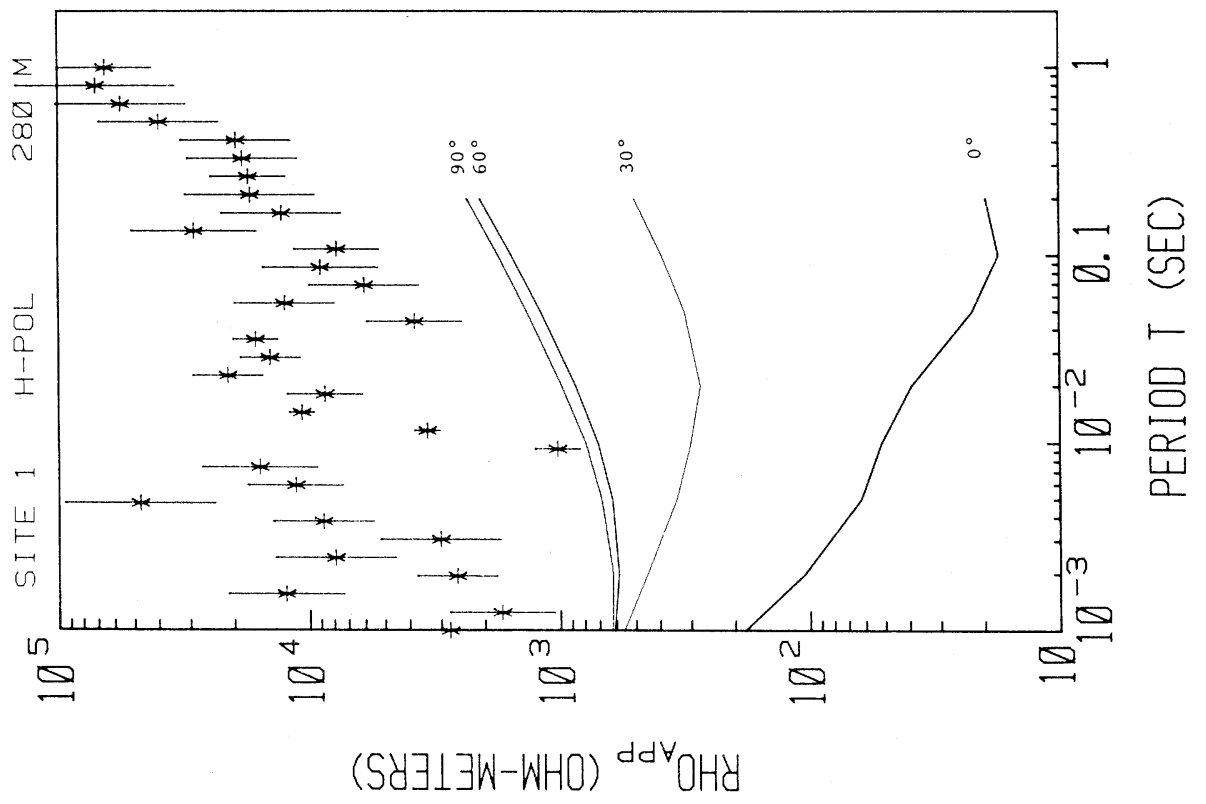

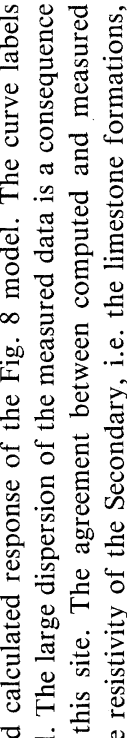

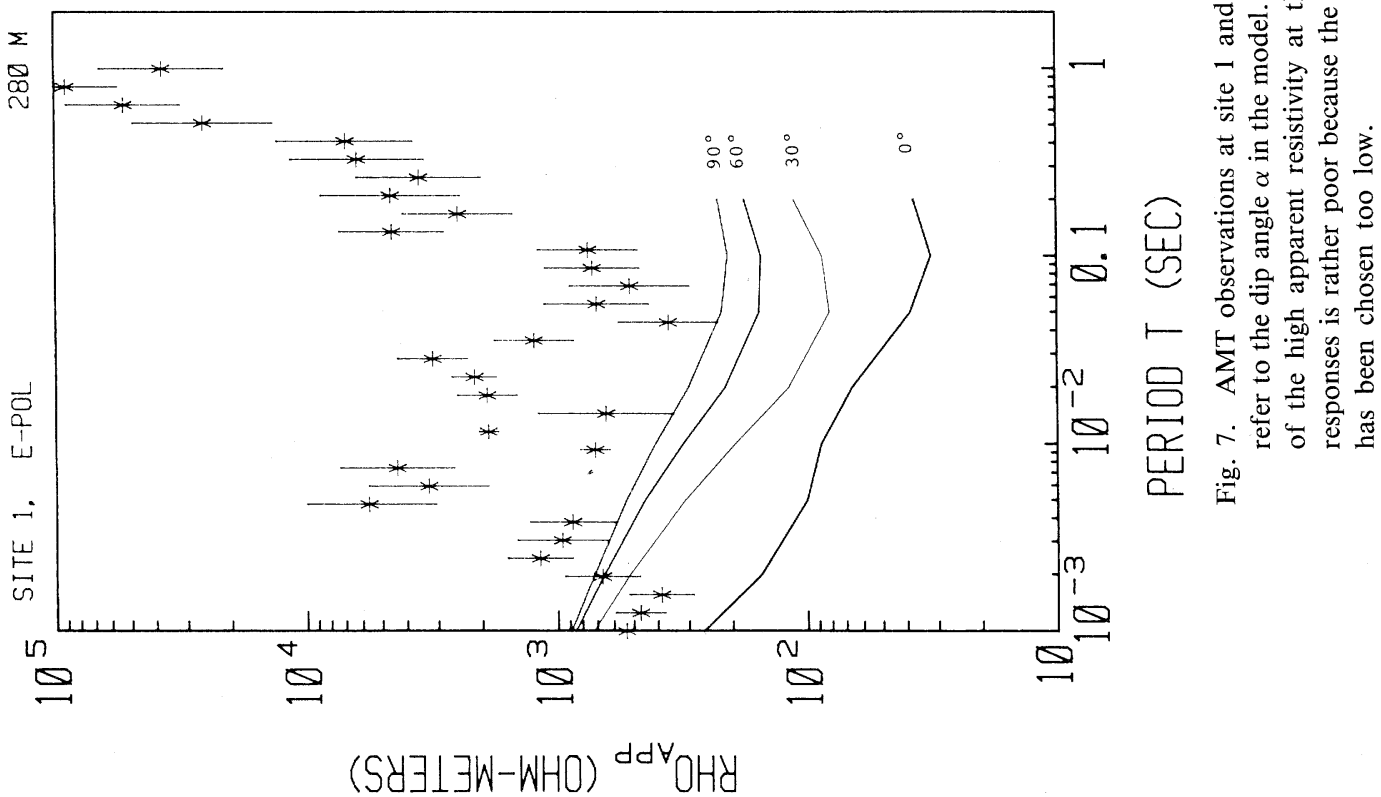




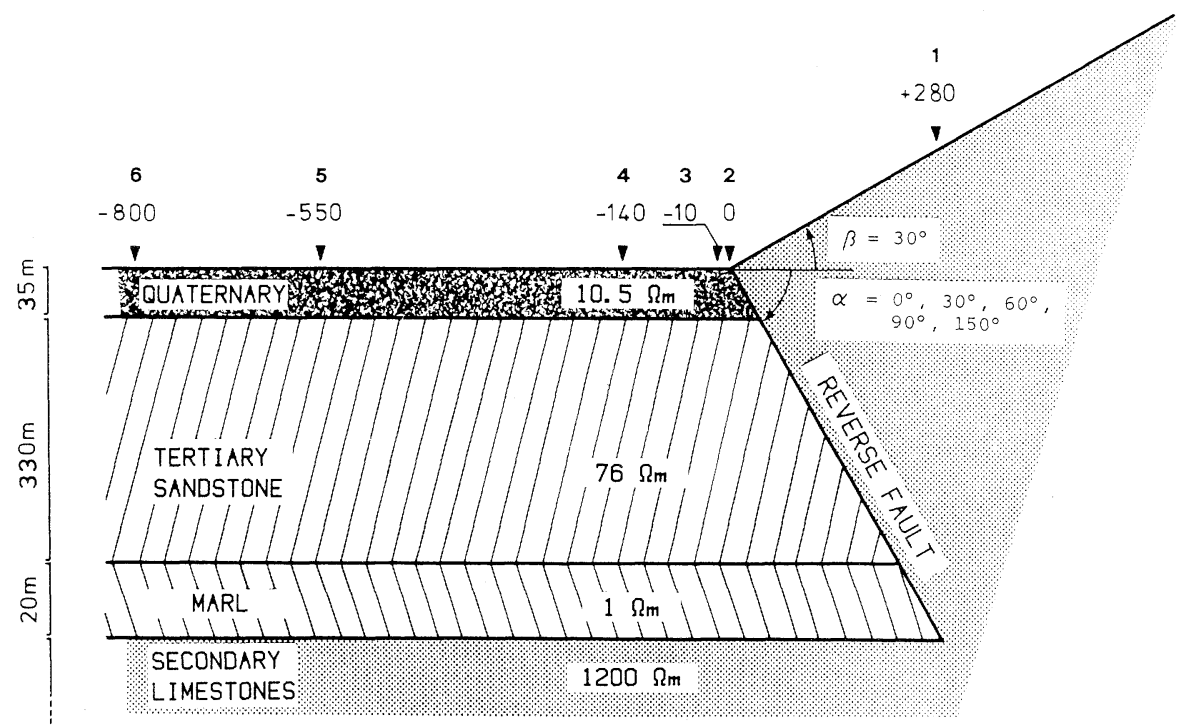

Fig. 8. Simplified 2-D model used for the numerical computation, where the 1-D valley structure is derived from the H-POL data at site 4 (cf. Fig. 9). In this 2-D model the only variable parameter is the dip angle $\alpha$ of the fault.

but little has been published about it, excepting one fairly complete description in some little-known conference proceedings (SCHNEGG and FISCHER, 1980). Several important details have been improved since that publication, however, making it worthwhile briefly to describe the system again. Our control electronics is not merely a data logger, but is built around a microcomputer so as to yield, in real time, preliminary apparent resistivities in the field. The heart of the microcomputer is a 16-bit Texas Instruments TMS 9900 microprocessor. The five AMT signals are first low-pass filtered at $1000 \mathrm{~Hz}$ and $125 \mathrm{~Hz}$, and notch-filtered to remove as much $162 / 3 \mathrm{~Hz}$ (the Swiss and German railway mains), $50 \mathrm{~Hz}$, and $150 \mathrm{~Hz}$ as possible. The two sets of analog signal are then digitized at frequencies of $4000 \mathrm{~Hz}$ and $250 \mathrm{~Hz}$. With these sampling frequencies and the lowpass filters mentioned above, all aliasing errors are safely avoided.

After a few minutes of sounding, the scope display of apparent resistivities enables the crew to decide whether the sounding is proceeding satisfactorily, or whether a less perturbed site should be sought. If the site is deemed suitable, 48 samples, each consisting of 1024 data points for each of the five channels and for each of the two digitizing frequencies are recorded on a standard magnetic tape. The tape format is such as to be readable by large computers for further processing. To execute a sounding requires about 45 minutes. This includes the time necessary to deploy the equipment and to collect it when the sounding is finished. The two truck batteries $( \pm 12 \mathrm{~V}, 120 \mathrm{Ah})$ last for 16 soundings before recharging is necessary. 



Fig. 9. H-POL measurements at site 4. The smooth curve is the response of a 1-D structure (cf. the Table or Fig. 8) obtained by modelling the apparent resistivity data points plotted. Modelling was carried out under the constraints that layer 4 has a resistivity of $1200 \Omega \mathrm{m}$ and layer 3 a thickness of $20 \mathrm{~m}$. These constraints were suggested by independent geological information, but the $1200 \Omega \mathrm{m}$ for layer 4 seems inadequate (cf. Fig. 7).

At AMT frequencies the electrodes simply consist of brass rods, $25 \mathrm{~m}$ apart. The French CM-16 (ECA) coils (ANDRIEUX et al., 1974) have been improved and now yield useful data from 1 to $1000 \mathrm{~Hz}$, instead of the original 8 to 400 $\mathrm{Hz}$. But the most important feature, in our view, which makes it possible at all to perform AMT soundings in Switzerland, is the delay-line filtering of the data (SCHNEGG and Fischer, 1980; Fischer, 1982). The level of artificial perturbations is so high in Switzerland that the remote magnetic reference technique of GAMBle et al. $(1979 \mathrm{a}, \mathrm{b})$ does not seem to work in the AMT range (cf. FISCHER, 1982), but delay-line filtering remains quite effective (it appears that the remote reference method requires a reference which is not, itself, too highly perturbed). However, high resistivity sites still present a problem, as mentioned in the Introduction. 


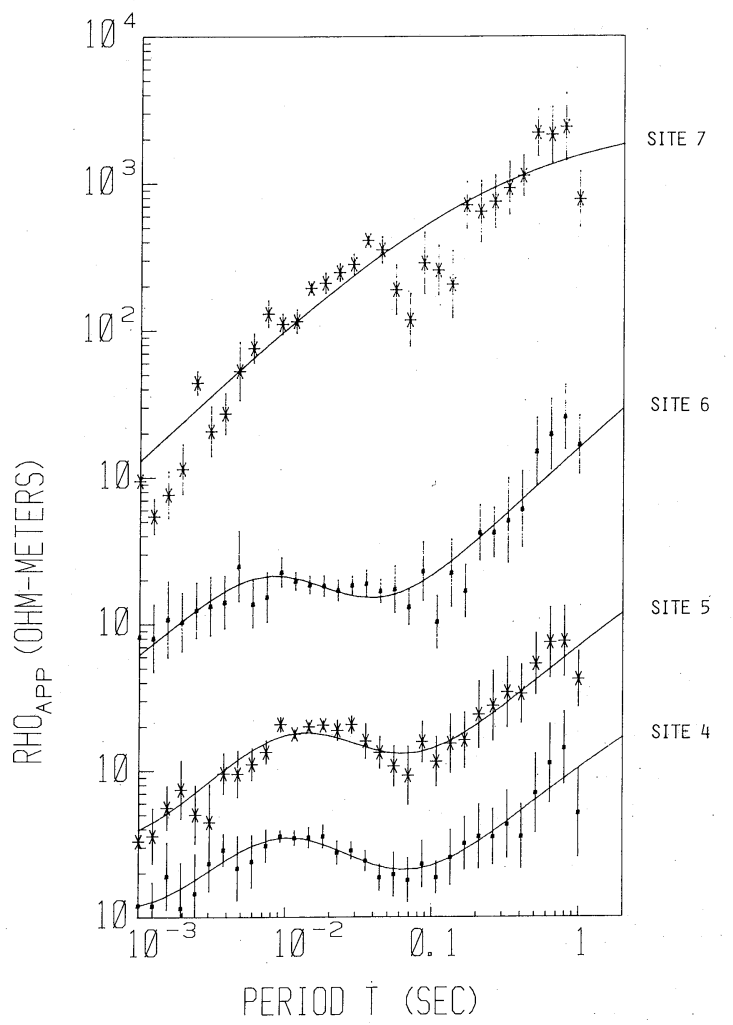

Fig. 10. AMT data from sites $4-7$ (H-POL), which suggest that the valley formations are dipping slightly toward the mountains (cf. the Table).

Along a profile of slightly more than one km length six soundings have been carried out, at the sites shown in Fig. 1 and 8. A control sounding was also carried out at a site $2 \mathrm{~km}$ further NW along the profile (site no. 7), where, according to the Swiss Geological Atlas, the transition between Tertiary and Secondary has been identified by seismic sounding as being at $120-130 \mathrm{~m}$ from the surface. Since all the other AMT soundings in the valley always returned four layers upon 1-D modelling (FISCHER and LE QUANG, 1981, 1982), the control sounding was intended to help deciding whether the transition to the high resistivity base was to be sought at the Secondary-Tertiary boundary or rather at a lower interface among the numerous secondary layers. As suggested by Table 1 and Figs. 3 and 10 our data support the first of these two alternatives, although this point will be discussed in some detail in Section. 7. It should be noted that sounding at site 1 on the $30^{\circ}$ mountain slope was performed parallel to the ground. This should be taken into consideration when comparing with model calculations, as discussed in Section 6. 
Table 1. Situation of the seven AMT sounding sites. For sites 4-7 the results of 1-D apparent resistivity modelling (FISCHER and LE QuANG, 1981) are given; site 7 only requires two layers (cf. Fig. 10).

\begin{tabular}{|c|c|c|c|c|c|c|c|c|}
\hline $\begin{array}{l}\text { Site/Pol. } \\
\text { No/E, H }\end{array}$ & $\begin{array}{c}\rho_{1} / h_{1} \\
\Omega \mathrm{m} / \mathrm{m}\end{array}$ & $\begin{array}{c}\rho_{2} / h_{2} \\
\Omega \mathrm{m} / \mathrm{m}\end{array}$ & $\begin{array}{c}\rho_{3} / h_{3} \\
\Omega \mathrm{m} / \mathrm{m}\end{array}$ & $\begin{array}{l}\rho_{\text {ult }} \\
\Omega \mathrm{m}\end{array}$ & $\begin{array}{l}\text { Depth to } \\
\text { resistor } \\
\mathrm{m}\end{array}$ & $\begin{array}{c}\text { Azimuth } \\
\text { with respect } \\
\text { to strike }\end{array}$ & $\begin{array}{l}\text { Dist. from } \\
\text { site } 2 \\
\mathrm{~m}\end{array}$ & $\begin{array}{c}\text { Altitude } \\
\mathrm{m}\end{array}$ \\
\hline $1 /-$ & - & - & - & $10^{3}-10^{4}$ & 0 & $+25^{\circ}$ & 280 & 960 \\
\hline $2 /-$ & - & - & - & $10^{3}-10^{4}$ & - & +24 & 0 & 823 \\
\hline $3 /-$ & — & - & - & $10^{3}-10^{4}$ & - & $+9^{\circ}$ & -10 & 821 \\
\hline $4 / \mathrm{H}$ & $10.5 / 35$ & $76 / 330$ & $1 / \underline{20}$ & $\underline{1200}$ & 365 & $+20^{\circ}$ & -140 & 818 \\
\hline $5 / \mathrm{H}$ & $4.3 / 30$ & $\underline{500} / 230$ & $3.6 / 100$ & $\underline{1200}$ & 360 & $+10^{\circ}$ & -550 & 788 \\
\hline $6 / \mathrm{E}$ & $3.0 / 15$ & $390 / 220$ & $6.5 \cdot 98$ & 1220 & 333 & $+8^{\circ}$ & -800 & 775 \\
\hline $6 / \mathrm{H}$ & $4.2 / \underline{20}$ & $\underline{300} / 183$ & $6.7 / 137$ & $10^{4}$ & 340 & $+8^{\circ}$ & -800 & 775 \\
\hline $7 / \mathrm{H}$ & $4.2 / 13$ & - & - & 2900 & 13 & $+20^{\circ}$ & -2500 & 722 \\
\hline
\end{tabular}

Underlined values have been fixed arbitrarily.

Except for the delay-line filtering the sounding data were analysed in rather standard fashion and the impedances were rotated into principal coordinates. In spite of the fact that all the sounding curves appeared to be highly isotropic, the azimuth returns and shown in the Table all fall close to the geological strike at about $\mathrm{N} 26^{\circ} \mathrm{E}$.

\section{AMT Sounding Results}

Figs. 4 to 7 show the E- and H-polarization sounding results at sites 6, 4, 2 and 1 respectively. The E-POL diagrams refer to electric polarization along the principal azimuth given in the Table. The sounding diagrams from sites 3 and 5 are very similar to those from site 4 .

Two features stand out in these diagrams: (1) at all the sites, except perhaps site 1, the apparent resistivity is highly isotropic, even though what has been plotted refers to the principal impedance coordinates which maximize the difference between the two resistivities (FISCHER, 1975); (2) the apparent resistivities at the valley sites 2 to 6 are almost identically low, whereas the resistivities at the mountain site 1 are very high.

In trying to explain these results the data from sites 2 and 3 play a crucial role. These two sites are at the base of the mountain. In the absence of a fault and of a conducting talus or scree, they would have to be looked at as being located on very resistive ground but immediately adjacent to a highly conducting formation. This is somewhat reminiscent of an ocean coast situation, and one would therefore expect a rather anisotropic impedance with a small apparent resistivity in the E-POL configuration and a large one for H-POL (cf, FISCHER et al., 1978; FISCHER, 1979; and RAVAL et al., 1981). The absence of any anisotropy is strong evidence by itself that there must be a fault as sketched in Fig. 3. 


\section{Interpretation in Terms of a 2-D Structure}

On the basis of these arguments the attempt was made to explain the data in terms of the simple model shown in Fig. 8. The horizontal layering at the left of this model was derived from 1-D modelling of the site 4 resistivity data (cf. FISCHER and LE QUANG, 1981). Because of a slightly larger scatter the decision was made not to model the phase, but the computed 1-D response curves of Fig. 9 show that the observed phase is nevertheless reasonably well accounted for by the 1-D model. Having chosen the horizontal layering in the valley, a simplified 2-D model based on the structure of Fig. 3 was designed, such as to be amenable to computation by the method of finite differences (see Section 6). This model is shown in Fig 8, in which it will be noted that all the structural parameters are fixed, with the exception of the angle of dip $\alpha$ of the fault. The 1-D model response will now be computed for various values of the unknown angle $\alpha$ and it is hoped that the comparison between calculated and measured data will permit the determination of the dip angle $\alpha$.

As the comparison between measured and computed data suggests, the decision to use the site 4 results to derive the 1-D valley structure was not the wisest. This site is fairly close to the mountain base and its response is not determined only by the 1-D valley structure, but also by the 2-D structural features of the mountain, as is evident from the computed model response in Fig. 5. Choosing site 6 would have been better justified.

We also note that in the absence of any fault the upward bending of the valley formations, as one moves toward the mountain, would be well described by setting $\alpha=150^{\circ}$. We have thus computed the response for this angle too, but the responses obtained are highly anisotropic and totally disagree with the observations; they have therefore not been plotted. As noted earlier, this observation alone is a strong case in favor of the existence of a fault.

Figure 4 displays measured and computed data from site 6 and we see that this site is almost unaffected by the presence of the mountain: the computed curves vary little when the dip angle $\alpha$ is varied. At site 4 (Fig. 5) this angle has already a greater influence on the computed results, but all the model curves are still too close together to permit a selection of $\alpha$. We also note the perfect accord when $\alpha=0$ between the H-POL curve and the measured data. Since these measurements had been selected to determine the 1-D valley structure this agreement confirms the validity of our 2-D model computations. The site 2 data from Fig. 6 are evidently the most critical for the choice of $\alpha$. Site 2 is exactly at the foot of the mountain and one would expect that the tilt of the fault should have a great importance in determining the response observed at this site. That the E-POL response is rather insensitive to $\alpha$ is understandable, since the tangential continuity of the electric fields tends to maintain this field at the low value characteristic of the good conductor. Under H-POL, however, there is continuity of the current, with large variations of the E-field at the site 2 vertex. Only the curves corresponding to $\alpha=30^{\circ}$ or $0^{\circ}$ agree with the measured data while 
the one for $\alpha=90^{\circ}$ even falls outside the range of the drawing, toward very large values of apparent resistivity. One should point out, however, that the computations at this particular site are somewhat more critical because of the large discontinuity in resistivity, the sharp angles of the structure and the changing slope of the surface topography. Furthermore, we must remember that the measurements were actually performed at the surface of the talus, a good conductor whose presence has been neglected in the model. This talus most certainly smooths out the effects of the various discontinuities just enumerated.

Finally, site 1 located half-way up the mountain, yields the data and curves of Fig. 7. Here the measured apparent resistivities are much higher than in the valley, because the limestones crop out to the surface. The larger dispersion of the data has been explained in Sec. 2 as a consequence of these high resistivities. Also apparent in Fig. 7 is that our model value for the limestone resistivity is clearly too low, which accounts for the poorer fit between data and curves. With a higher limestone resistivity of about $4000 \Omega \mathrm{m}$ in the model of Fig. 8 a better fit would have been achieved in Fig. 7, without noticeably affecting the agreement obtained at the other sites. Nevertheless, it is clear that a dip angle $\alpha$ of $30^{\circ}$ is more compatible with the site 1 data, than a dip angle of $0^{\circ}$, and in view of the highly selective choice required by the site 2 results we conclude that the existence of a reverse fault with an angle of dip of $30^{\circ}$ or somewhat less is fairly well established.

\section{Numerical Modelling}

The two-dimensional model response was computed by the method of finite differences developed by BREWITT-TAYLOR and WEAVER (1976) with the improved boundary conditions (WEAVER and BREWITT-TAYLOR, 1978) incorporated in the E-polarization calculations.

The method was originally developed on the assumption that the surface of the Earth was a horizontal plane and that there were no regions of vanishing conductivity beneath this plane. Thus in order to model properly the topography of the Val de Ruz region it was necessary to take the plane $z=0$ at the level of the top of the mountain slope ( $314 \mathrm{~m}$ above the valley floor) and to assign a very low, but non-vanishing, conductivity value for the region between this level and the actual surface of the sloping ground. It was found that a value of $10^{-19} \mathrm{~S} / \mathrm{m}$ was quite effective; it caused no problems with the finite difference calculations and gave results that were consistent with the behaviour of an electromagnetic field in a perfect insulator. For example, it is well known that an $\mathrm{H}$-polarization magnetic field in a non-conducting region is everywhere constant (usually taken to be unity) and the finite difference calculations did indeed yield a constant unit magnetic field in the region below $z=0$ where the conductivity was given the very small value.

Calculations at the site on the sloping mountain side also required some special consideration. In a finite difference model the slope must be approx- 
imated by a "staircase" whose grid points have conductivities (resistivities in H-polarization) given by the weighted means of the values assigned to the four neighbouring cells. The field components at a site on the slope are therefore most sensibly calculated at a grid point in the middle of one of the horizontal "steps" of the staircase, removed from any sharp corners. The field components that are tangential to the (local) ground surface at such a point are the usual horizontal components given by the finite difference calculations but they are not the components parallel to the sloping ground, one up the slope and the other horizontally transverse to the slope, as measured in the field. However, it was decided not to attempt to convert the calculated results into the directions of these latter components because in $\mathrm{H}$-polarization this would have entailed calculating the vertical as well the horizontal electric field on the surface of the "step" and this is not readily done in a very poorly conducting region. Moreover, it is not clear that a field component parallel to the overall direction of the slope, but not tangential to the local ground surface in the model, would provide a realistic estimate of the actual apparent resistivity and phase measured in the field. Nevertheless these differences in local directions should be borne in mind when comparing the computed values with the field data obtained at a station on the mountain slope.

In the original version of the finite difference calculation (BREWITT-TAYLOR and WEAVER, 1976) the derived fields (the magnetic components in E-polarization, the electric components in H-polarization) were computed by central differences at the centre of the grid cells rather than at the grid points. Since our primary concern in this paper is to compute apparent resistivities it is clearly imperative to obtain the derived horizontal components at the grid points themselves on the surface of the Earth. Special formulae were quoted by Brewitt-Taylor and Weaver (their equations 6.10 and 7.9) giving these components at the grid points on $z=0$, and it is easily shown that the same formulae continue to hold at any grid point situated on a locally flat, horizontal portion of the Earth's surface, wherever it may be situated in the numerical model, such as on one of the "steps" in the "staircase" defining the mountain slope. These formulae were used in the apparent resistivity and phase calculations.

As remarked in Section 5 the calculation of the apparent resistivity at the vertex of the fault is extremely sensitive in the H-POL mode, changing from quite large values on the right of the vertex to much smaller values on the left. For consistent results, the numerical models were designed so that Site 2 was always at the grid point on the dividing line between the cells of different resistivities $-10.5 \Omega \mathrm{m}$ on the left and $1200 \Omega \mathrm{m}$ on the right. In this way, the apparent resistivity calculated at Site 2 was effectively a weighted average of the values at the neighbouring grid points on either side of the vertex.

\section{Detailed Investigation of the Valley Structure}

The data from the valley sites 4 to 7 have all been modelled with the 1-D 
routine and yield the results listed in Table 1. It seems that the mesozoic or secondary structure is dipping slightly in front of the fault, as would be expected if this structure had arisen through lateral compression. This conclusion is even more apparent in Fig. 10 which suggests indeed that the depth to the resistor, i.e. to the top of the secondary formations, occurs at increasing levels. As seen in the Table, however, this increase in depth is partly compensated by the rise in altitude, so that the dip of secondary and tertiary formations is probably no more than $5^{\circ}$.

The interpretation of MT or AMT soundings requires a great deal of care. While FISCHER and LE QUANG $(1981,1982)$ have shown that a given data set generally leads unequivocally to a single best-fitting 1-D structure, they have also given evidence that the various structural parameters $\rho_{i}$ and $h_{i}$ are obtained with widely varying degrees of confidence. To illustrate the meaning of this statement, one may ask about the range of values that a particular structural parameter may assume if one tolerates a $10 \%$ increase of the standard deviation $\varepsilon$ between measured and calculated MT response parameters. The solid lines in Fig. 11 show the admissible parameter ranges when a single parameter is varied. The dashed contours in this figure show that the ranges can be increased when the other parameters are simultaneously adjusted to minimize $\varepsilon$ under the constraint of a single imposed parameter change. While Fig.11 makes it obvious that in some cases wide parameter variations are possible, it must be kept in mind that these ranges are much reduced here since the adjacent soundings 4 to 6 must be made to agree with each other as well as with the known geological facts. These known factors are (1) that the top Quaternary is a good conductor, (2) that the underlying Tertiary consists of a sandstone bed over $100 \mathrm{~m}$ thick, often underlain by a thin bed of good conducting marls up to $20 \mathrm{~m}$ thick, sometimes containing inclusions of gypsum. The next formation is the mesozoic Secondary, a succession of high resistivity limestones with occasional thin layers of better conducting marls. But these secondary marls are too thin and not conducting enough to be assimilated with the good third conductor that we see at sites 2 to 6 . This good third conductor must instead by identified as being the thin tertiary marl basement. While the best fitting 1-D models suggest thicknesses around $100 \mathrm{~m}$, it is easy to verify that the only definite pronouncements that our data allow, is that the resistivity of this third layer is less than $18 \Omega \mathrm{m}$ and that its integrated conductivity, i.e. the ratio $h_{3} / \rho_{3}$, is about 20 mho. This means that an equally good fit can be achieved with $100 \mathrm{~m}$ at $5 \Omega \mathrm{m}$, or $20 \mathrm{~m}$ at $1 \Omega \mathrm{m}$. As far as the second layer, the tertiary sandstone, is concerned Fig. 11 shows that its thickness is determined with a greater degree of confidence and lies in the range between 180 and 600 meters. In fact, if we take advantage of the known range of these sandstone resistivities $(50-100 \Omega \mathrm{m})$, the extent of the probable sandstone thicknesses is narrowed, becoming then $300-450 \mathrm{~m}$.

The response observed at site 7 can be accounted for with two layers only, a thin good conductor on top and a resistive base. The upper conductor is again the Quaternary, reduced at site 7 to a thickness of only $10-20 \mathrm{~m}$. The resistor 

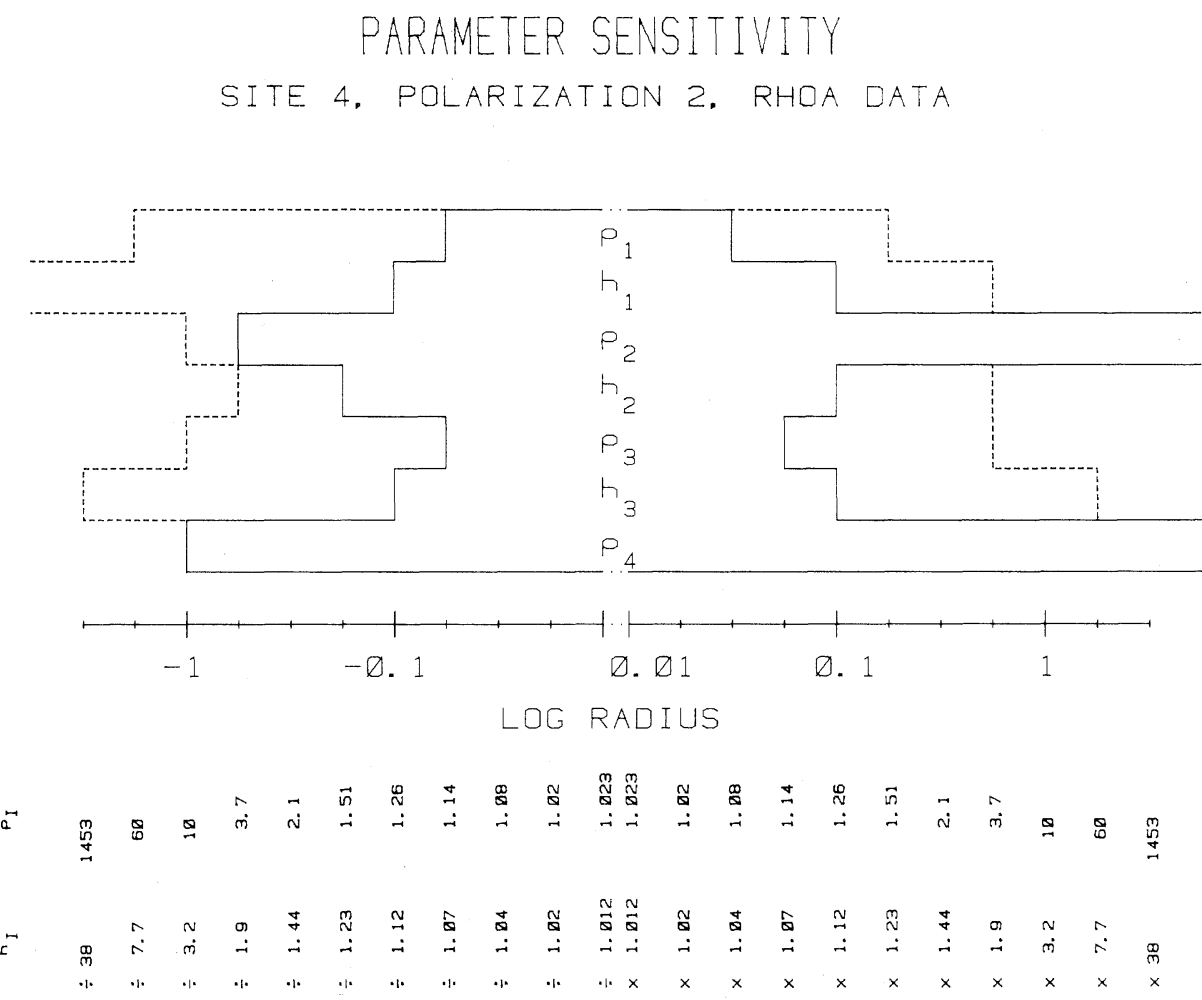

Fig. 11. Trade-off diagram of the model parameters obtained when modelling the site 4 apparent resistivity data (FISCHER and LE QUANG, 1981, 1982).

underneath is formed by the juxtaposition of tertiary sandstone, here only 110 - $120 \mathrm{~m}$ thick according to the seismic sounding mentioned in Sec. 3, and secondary limestones. This implies that the thin tertiary marls, obviously present at sites 2-6, are absent at site 7 . This is not surprising since these tertiary marl formations are known to be discontinuous in the Val de Ruz area. Without the intervening third good-conducting layer the AMT method is unable separately to identify the rather thin sandstone layer on top of the thick limestone base.

If we believe that the sandstone formation has indeed a thickness of at least $300 \mathrm{~m}$ at the base of the mountain, then the thickness of the Tertiary must decrease as one moves to the NW along the profile, and the top of the Secondary will probably be inclined between $3^{\circ}$ and $5^{\circ}$.

\section{Conclusions}

In spite of the difficulties inherent in the use of the AMT technique in areas with a high level of perturbation, this technique has proved well suited to in- 
vestigate a specific 2-D feature. The success of the present study must of course be attributed to the fact that this 2-D feature, a geological reverse fault, involves high contrasts of resistivity. But high conductivity contrasts are almost a prerequisite in electrical or electromagnetic soundings. Other important helpful features were the clear geological constraints. The AMT method worked because it only had limited questions to answer. It is doubtful whether any definitive answers could have been obtained in the absence of reliable prior geological information.

Given these geological constraints the AMT method was not only capable of identifying the presence of a fault, but it also succeeded in determining its angle of dip, as well as the structure in front of the fault.

This research project received support from the authors' institutions, as well as from the Swiss National Science Foundation, The Geological Commission from the Swiss Academy of Natural Sciences, and the Natural Sciences and Engineering Research Council of Canada. Help in computational work by E.L. Friesen is also gratefully acknowledged.

\section{REFERENCES}

BREWITT-TAYLOR, C. R. and P. B. Johns, Diakoptic solution of induction problems, J. Geomag. Geoelectr., 32 Suppl. I, SI 73-SI 78, 1980.

BREWITT-TAYLOR, C. R. and J. T. WEAVER, On the finite difference solution of two-dimensional induction problems, Geophys. J. R. Astr. Soc., 47, 375-396, 1976.

Andrieux, P., G. ClerC, and P. TORT, Capteur magnétométrique triaxial pour la prospection magnétotellurique artificielle entre $4 \mathrm{~Hz}$ et $4 \mathrm{kHz}$, Rev. Physique Appl., 9, 757-759, 1974.

FISCHER, G., Symmetry properties of the surface impedance tensor for structures with a vertical plane of symmetry, Geophysics, 40, 1046-1050, 1975.

FISCHER, G., Electromagnetic induction effects at an ocean coast, Proc. IEEE, 67, 1050-1060, 1979.

FISCHER, G., Magnetotelluric observational techniques on land, Geophys. Surv., 4, 373-393, 1982.

FISCHER, G., P. -A. SCHNEGG, and K. D. USADEL, Electromagnetic response of an ocean-coast model to E-polarization induction, Geophys. J. R. Astr. Soc., 53, 599-616, 1978.

FISCHER, G. and B. V. LE QUANG, Topography and minimization of the standard deviation in one-dimensional magnetotelluric modelling, Geophys. J. R. Astr. Soc., 67, 279-292, 1981.

FISCHER, G. and B. V. LE QUANG, Parameter trade-off in one-dimensional magnetotelluric modelling, J. Geophysics/Z. Geophysik, 51, 206-215, 1982.

Gamble, T. D., W. M. Goubau, and J. Clarke, Magnetotellurics with a remote magnetic reference, Geophysics, 44, 53-68, 1979a.

Gamble, T. D., W. M. Goubau, and J. Clarke, Error analysis for remote reference magnetotellurics, Geophysics, 44, 959-968, 1979b.

Hutton, V. R. S., M. R. Ingham, and E. W. MBIPOM, An electrical model of the crust and upper mantle in Scotland, Nature, Eond., 287, 30-33, 1980.

Jupp, D. L. B. and K. Vozoff, Two-dimensional magnetotelluric inversion, Geophys. J. Roy. Astr. Soc., 50, 333-352, 1977.

RAVAl, U., J. T. WeAVER, and T. W. DAwSOn, The ocean-coast effect re-examined, Geophys. J. Roy. Astr. Soc., 67, 115-123, 1981.

SCHNEGG, P. -A. and G. FISCHER, On-line determination of apparent resistivity in magnetotelluric sounding, Protokoll Kolloquium Elektromagnetische Tiefenforschung (Berlin-Lichtenrade, April 1-3, 1980), pp. 173-184, 1980.

Swiss Geological Atlas, Map and Explanatory Notices no. 51/1144, Val de Ruz, 1969. 
Stanley, W. D., J. E. Boehl, F. X. Bostick, and H. W. Smith, Geothermal significance of magnetotelluric sounding in the eastern Snake-River plain - Yellowstone region, J. Geophys. Res., 82, 2501-2514, 1977.

WEAVER, J. T. and C. R. BREWITT-TAYLOR, Improved boundary conditions for the numerical solution of E-polarization problems in geomagnetic induction, Geophys. J. R. Astr. Soc., 54, 309-317, 1978. 